Propriétés amphiphiles des monolaurates de polyglycérol: application à la préparation de microémulsions à base d'esters gras

Oléagineux, Corps Gras, Lipides. Volume 10, Numéro 5-6, 382-6, Double 5-6, SEPTEMBRE-OCTOBRENOVEMBRE-DÉCEMBRE 2003, UTILISATIONS ET APPLICATIONS DES MATIÈRES PREMIÈRES RENOUVELABLES

Auteur(s) : Nelly CHAILLOUX, Véronique NARDELLO, Jean-Louis SALAGER, Jean-Marie AUBRY, LCOM, Equipe de Recherche "Oxydation \& Formulation", UMR CNRS 8009, ENSCL, F59652 Villeneuve d'Ascq cedex, France 03.20.33.63.69 < veronique.ratajuniv-lille1.fr> Laboratoire FIRP, Ingenieria Quimica, Universidad de Los Andes, Merida, 5101, Venezuela .

Author(s) : Nelly CHAILLOUX, Véronique NARDELLO, Jean-Louis SALAGER, Jean-Marie AUBRY

Résumé : Les esters de polyglycérol des acides gras sont des tensioactifs non ioniques, d'origine entièrement végétale, et totalement biodégradables. Le comportement de phase des systèmes eauhuile-monolaurate de polyglycérol a été étudié par le concept du Hydrophilic Lipophilic Deviation. Ce concept a permis de caractériser les tensioactifs de la famille du monolaurate de polyglycérol $C_{12} G_{j}$ et d'établir une classification de diverses huiles de type ester selon leur valeur de EACN (Equivalent Alkane Carbon Number).

Summary : Polyglycerol fatty esters are non ionic, surfactants, based on vegetable oils and totally biodegradable. The phase behaviour of the water-oil-polyglycerol monolaurate systems was studied by the HLD concept or Hydrophilic Lipophilic Deviation. This concept allowed to characterize the surfactants of the polyglycerol monolaurate family, noted $\mathrm{C}_{12} \mathrm{G}_{\mathrm{j}}$, and to establish a classification of several ester oils according to their EACN value (Equivalent Alkane Carbon Number).

Mots-clés : monolaurate de polyglycérol, microémulsion, ester gras, nombre équivalent d'atome de carbone, EACN, différence hydrophile lipophile, HLD

Keywords : polyglycerol monolaurate, microemulsion, fatty ester, equivalent alkane carbon number, EACN, hydrophilic lipophilic deviation, HLD

\title{
ARTICLE
}

Auteur(s) : Nelly CHAILLOUX ${ }^{1}$, Véronique NARDELLO ${ }^{1 *}$, Jean-Louis SALAGER ${ }^{2}$, Jean-Marie AUBRY ${ }^{1}$

${ }^{1}$ LCOM, Equipe de Recherche "Oxydation \& Formulation », UMR CNRS 8009, ENSCL, F-

59652 Villeneuve d'Ascq cedex, France 03.20.33.63.69

<veronique.rataj@univ-lille1.fr>

${ }^{2}$ Laboratoire FIRP, Ingenieria Quimica, Universidad de Los Andes, Merida, 5101, Venezuela

Les esters de polyglycérol, notés $C_{i} G_{j}$, sont des tensioactifs non ioniques d'origine entièrement végétale, inodores et totalement biodégradables. Ils sont obtenus par estérification d'acides gras, 
issus des triglycérides d'huiles végétales, sur un polyglycérol, obtenu par polymérisation catalytique du glycérol en milieu alcalin [1-3]. Par conséquent, les esters de polyglycérol commerciaux sont constitués d'un mélange complexe de composés, dépendant du degré de polymérisation du glycérol, des isomères de polyglycérol, du nombre d'acides ayant réagi par molécule de tensioactif et du type d'acide gras estérifié. Les esters de polyglycérol trouvent leurs principales applications dans les domaines alimentaire, cosmétique et pharmaceutique [4-6]. Ce sont en effet des produits présentant (i) une excellente tolérance pour la peau, (ii) une meilleure stabilité thermique que les autres tensioactifs non ioniques traditionnels et (iii) une activité antimicrobienne modérée. De plus, la partie lipophile, constituée d'un acide gras, combinée à une partie hydrophile à base de glycérol, leur confère, en plus de leurs propriétés tensioactives, des propriétés émolliente et hydratante. Toutes ces propriétés, associées au contexte actuel où de nombreux facteurs économiques (prix élevé de l'éthylène), psychologiques (syndrome de l'encéphalopathie spongiforme bovine, intérêt des consommateurs pour les produits d'origine végétale), environnementaux (faible biodégradabilité des chaînes ramifiées ou aromatiques d'origine pétrolière) et réglementaires (interdiction des alkylphénols éthoxylés), concourent à favoriser le développement de ce type de tensioactif. Par ailleurs, il a souvent été constaté que des mélanges appropriés de tensioactifs permettent d'obtenir des performances en application meilleures que celles des tensioactifs purs en ce qui concerne l'abaissement des tensions superficielles ou interfaciales, la solubilisation d'huile et d'eau en microémulsion ou encore la stabilité des émulsions et des mousses [7]. II a ainsi été établi que des mélanges d'alcools gras polyoxyéthylénés de longueur de chaîne variable améliorent considérablement la solubilisation $d^{\prime}$ huile et d'eau dans les microémulsions bicontinues [8,9]. Dans ce contexte, il nous est apparu intéressant d'étudier le comportement de phase des esters de polyglycérol dans les systèmes ternaires eau-huile-tensioactif en recourant au concept du HLD (différence hydrophile lipophile). Le HLD, qui est une version moderne du paramètre HLB caractéristique des tensioactifs, inclut les effets des autres variables physico-chimiques comme la nature de I'huile, la température, la salinité Cet outil permet de quantifier le comportement de phase d'un système à l'aide d'une seule variable de formulation et de caractériser les huiles et les tensioactifs [10-15]. En effet, les influences relatives de chacun des paramètres intervenant sur l'équilibre hydrophile/lipophile du système peuvent être quantifiées et des échelles absolues de classification des huiles ou des tensioactifs peuvent être établies. Nous avons choisi d'étudier des tensioactifs de la famille du monolaurate de polyglycérol, notés $C_{12} G_{j}$, (1), en prenant comme variable de formulation le nombre moyen de motifs glycérol :

monolaurates de polyglycérol $\mathrm{C}_{12} \mathrm{G}_{\mathrm{j}}(1)$

\section{Partie expérimentale}

\section{Produits}

Les monolaurates de polyglycérol $\left(\mathrm{C}_{12} \mathrm{G}_{\mathrm{j}}\right)$ avec un nombre moyen de motifs glycérol égal à 4 $\left(\mathrm{C}_{12} \mathrm{G}_{4}\right), 6\left(\mathrm{C}_{12} \mathrm{G}_{6}\right)$ ou $10\left(\mathrm{C}_{12} \mathrm{G}_{10}\right)$ ont été fournis par la société Rossow (Paris). Les alcanes (99\%) (heptane, décane, dodécane, hexadécane), les esters éthyliques des acides en $\mathrm{C} 2, \mathrm{C} 6, \mathrm{C} 7, \mathrm{C}$, 
C10, C14 et l'éthanoate d'hexyle ( $99 \%)$, le propanoate de pentyle ( $99 \%)$, le butyrate de butyle (98\%), I'heptanoate de méthyle (99\%), le 1-hexanol (98\%) et le 2-butanol (99,5\%) ont été obtenus chez Aldrich. De l'eau distillée possédant une résistivité de $18,2 \mathrm{M} \Omega / \mathrm{cm}$ a été utilisée pour toutes les expériences.

\section{Méthodes}

\section{Balayages de formulation}

Un balayage unidimensionnel consiste à réaliser différents systèmes ternaires eau-huiletensioactif ayant la même composition à l'exception de la variable choisie pour effectuer le balayage (dans le cas présent, le nombre de motifs glycérol GN). Chaque système contient des volumes égaux d'huile et d'eau et $15 \%$ en poids de monolaurate de polyglycérol C12Gj. Dans certains cas, un co-tensioactif tel que du 2-butanol peut être ajouté à raison de $3 \%$ en poids pour accélérer la mise en équilibre du système sans modifier sa valeur de HLD [13]. Les systèmes sont maintenus quelques jours à $55^{\circ} \mathrm{C}$ jusqu'à ce que l'équilibre soit atteint. Le type de comportement de phase d'après Winsor peut être facilement déterminé en colorant la phase aqueuse en bleu à l'aide d'un colorant hydrosoluble et la phase huileuse en jaune à l'aide d'un colorant liposoluble; ainsi, la microémulsion se colore naturellement en vert. On trouvera de plus amples détails sur la manière de procéder et d'interpréter les résultats dans la littérature [10-15].

\section{Détection de l'optimum de formulation}

Le paramètre de solubilisation (PS) est défini comme étant le volume d'eau ou d'huile solubilisé dans la microémulsion par gramme de tensioactif. L'optimum de formulation est défini par $\mathrm{PS}_{\text {huile }}=\mathrm{PS}_{\text {eau }}$ et correspond au minimum de tension interfaciale d'après les travaux de $\mathrm{C}$. Huh [16].

\section{Résultats et discussion}

\section{Analyse des monolaurates de polyglycérol $C_{12} G_{j}$}

De façon générale, les esters de polyglycérol d'acides gras $C_{i} G_{\jmath}$ sont des mélanges complexes d'oligomères car le nombre de motifs glycérol, le nombre de substitution de la chaîne polyglycérol et la structure de la chaîne sont difficilement maîtrisés lors de la synthèse. En conséquence, peu de données analytiques sur ce type de tensioactifs sont disponibles dans la littérature. La plupart des études chromatographiques réalisées se limitent à un niveau qualitatif [17]. Néanmoins, quelques analyses quantitatives effectuées par couplage LC/MS ou GC/MS ont été décrites récemment [18]. Dans le cas des monolaurates de polyglycérol, une analyse par spectrométrie de masse a montré que la partie hydrophobe du tensioactif (issue de l'acide laurique) est relativement pure (>99\%). En revanche, la partie hydrophile, obtenue par condensation de motifs glycérol, est de longueur très variable. À titre d'exemple, le composé vendu en tant que monolaurate de tétraglycérol, $\mathrm{C}_{12} \mathrm{G}_{4}$ contient toute une série d'oligomères 
comportant un nombre de motifs glycérol allant de 1 à 10. Par ailleurs, le chromatogramme met également en évidence des polyglycérols non estérifiés comportant de 1 à 8 motifs glycérol.

\section{Le concept du HLD [8]}

Selon leur formulation, les systèmes ternaires eau-huile-tensioactif peuvent présenter, à l'équilibre, une ou plusieurs phases: une microémulsion monophasique (Winsor IV), une microémulsion en équilibre avec une phase huileuse (Winsor I) ou une phase aqueuse (Winsor II), une microémulsion en équilibre à la fois avec une phase huileuse et une phase aqueuse (Winsor III). Comme le montre la figure 1, les transitions de phase entre les différents systèmes de Winsor peuvent être mises en évidence, pour une concentration donnée en tensioactif, à l'aide d'une variable de formulation qui modifie l'hydrophilie ou l'hydrophobie du système eau-huiletensioactif (GN ou nombre de motifs glycérol dans le cas présent). La figure 1 indique que, pour des valeurs de $G N<5$ (faible hydrophilie), le comportement de phase est de type Winsor II, tandis que pour $\mathrm{GN}>7$, il est de type Winsor I. Pour une hydrophilie intermédiaire, on observe soit un comportement de phase de type Winsor III à faible concentration de tensioactif, soit un comportement de phase Winsor IV quand le système contient suffisamment de tensioactif (>10\%) pour co-solubiliser la totalité de l'eau et de l'huile. Enfin, on constate qu'à très faible concentration de tensioactif (on devrait plutôt dire «trop faible »), on n'a pas de microémulsion et on observe le passage direct du type Winsor II au type Winsor I. Dans le cas d'un système triphasique (Winsor III), la formulation est dite "optimale " si la microémulsion solubilise des quantités égales d'eau et d'huile. Cette formulation optimale est alors choisie comme origine pour établir une échelle absolue de classification des systèmes eau-huile-tensioactif indépendamment de leur composition. Cette échelle est basée sur la notion de HLD qui exprime quantitativement l'écart entre une formulation quelconque et la formulation optimale. Contrairement au HLB, concept empirique mais très largement utilisé, le HLD tient compte de toutes les variables de formulation: nature du tensioactif et de l'huile, type et quantité d'électrolyte et d'alcool, température. Le HLD est un nombre sans dimension, défini, dans le cas des tensioactifs non ioniques polyoxyéthylénés, par l'expression numérique suivante: $\mathrm{HLD}=\alpha-\mathrm{EON}+b \times \mathrm{S}-k \times \mathrm{ACN}-m \times \mathrm{A}+c_{T} \times \Delta \mathrm{T}(1)$ où $a, k, c_{T}$ dépendent de la structure chimique du tensioactif, EON est le nombre moyen de motifs oxyde d'éthylène par molécule de tensioactif, $S$ et $A$ représentent respectivement la concentration en pourcentage massique de sel et d'alcool, ACN est le nombre d'atomes de carbone de l'alcane linéaire, $\Delta T$ est la différence de température par rapport à $25^{\circ} \mathrm{C}, b$ est une constante dépendant du sel et $m$ une constante caractéristique de l'alcool et du tensioactif.

\section{Caractérisation des monolaurates de polyglycérol par la méthode du HLD}

La caractérisation d'un tensioactif au moyen du concept du HLD consiste à déterminer les constantes de l'équation (1) relatives au tensioactif. Dans le cas d'un ester de polyglycérol, le terme EON est alors remplacé par le terme GN représentant le nombre moyen de motifs glycérol par molécule de tensioactif. Dans un premier temps, il s'agit de déterminer la constante $k$, coefficient du terme ACN relatif à la longueur de chaîne de l'alcane. Pour ce faire, des balayages bidimensionnels sont réalisés pour différents alcanes linéaires dont le ACN varie de 7 à 16. La variable de balayage retenue pour l'étude du monolaurate de polyglycérol est le nombre de motifs glycérol, GN, obtenu en mélangeant dans des proportions appropriées les tensioactifs 
commercialement disponibles $\left(\mathrm{C}_{12} \mathrm{G}_{4}, \mathrm{C}_{12} \mathrm{G}_{6}\right.$ et $\left.\mathrm{C}_{12} \mathrm{G}_{10}\right)$ ayant une longueur de chaîne hydrophile variable. Les différents mélanges sont réalisés à $55^{\circ} \mathrm{C}$ afin de faciliter la dissolution des tensioactifs. Dans tous les cas, le rapport des volumes d'eau et d'huile est égal à 1 . La concentration en tensioactif est fixée à $15 \%$ en poids. En fonction des huiles étudiées (alcanes ou esters), les transitions observées sont WI $\rightarrow$ WIII $\rightarrow$ WII ou WI $\rightarrow$ WIV $\rightarrow$ WII. Lorsqu'elles mettent en jeu les systèmes Winsor III, la formulation optimale est fixée au point où les paramètres de solubilisation (PS) de l'eau et de l'huile dans la microémulsion sont exactement égaux. Dans le cas des transitions WI $\rightarrow$ WIV $\rightarrow$ WII, la formulation optimale est prise au centre de la zone de comportement monophasique Winsor IV. Avec les alcanes linéaires, seules les transitions WI $\rightarrow$ WIV $\rightarrow$ WII sont observées. On notera que les monolaurates de polyglycérol permettent d'obtenir des microémulsions dans la zone Winsor IV sans ajout de cotensioactif, probablement grâce à leur composition complexe qui apporte un certain désordre conformationnel et évite la formation de mésophases. Dans ces conditions et en l'absence de sel et d'alcool co-tensioactif, l'équation (1) devient (2) :

HLD $=$ Cste-GN-k $\times$ ACN (2)

La figure 2 représente l'évolution de GN*, valeur moyenne du nombre de motifs glycérol permettant d'obtenir la formulation optimale, en fonction du nombre d'atomes de carbone de l'alcane.

On constate que le nombre de motifs glycérol à la formulation optimale, GN*, décroît linéairement quand le ACN de l'alcane augmente. A la formulation optimale, nous pouvons donc écrire pour le monolaurate de polyglycérol :

$\mathrm{HLD}=0=6,53-\mathrm{GN}^{*}-0,10 \times \mathrm{ACN}(3)$

Précisons que des balayages similaires en température réalisés sur les systèmes à base de décane n'ont mis en évidence aucune influence de la température sur la formulation optimale, ce qui implique que, dans le cas des monolaurates de polyglycérol, la constante $c T$ est égale à 0 . Ce résultat, peu habituel pour des tensioactifs non ioniques, est en accord avec les observations de Kunieda et al. qui ont montré que le comportement de tensioactifs glycérylés est indépendant de la température [19]. D'autre part, il est bien connu que les alkylpolyglucosides, tensioactifs dont le groupe hydrophile est composé de fonctions hydroxyles, sont très peu sensibles à la température [20] contrairement aux alcools gras polyoxyéthylénés. En effet, ceci confirme que ce n'est pas le caractère non ionique du tensioactif qui le rend sensible à la température, mais la présence de fonctions éther.

\section{Comparaison des motifs glycérol et oxyde d'éthylène}

Les alcools gras polyoxyéthylénés, notés CiEj, sont les tensioactifs non ioniques les plus utilisés à I'heure actuelle et ils ont fait l'objet d'un nombre considérable d'études. L'application du concept du HLD à ces tensioactifs, notamment aux alcools lauriques $C_{12} E_{j}$, a conduit à la relation suivante applicable à la formulation optimale [13] :

$\mathrm{HLD}=5,9-\mathrm{EON}^{*}-0,15 \times \mathrm{ACN}(4)$

On constate que dans les équations (3) et (4), la constante $k$, caractéristique du tensioactif, est égale à 0,15 dans le cas des $C_{12} E_{j}$ tandis qu'elle est de 0,10 pour les $C_{12} G_{n}$. Cette différence est 
due au choix arbitraire du coefficient unitaire devant $\mathrm{GN}^{*}$ dans l'équation (3). Si l'on veut pouvoir comparer l'hydrophilie des groupes polaires, il est indispensable de les exprimer dans une même unité. II suffit pour cela d'écrire les équations (3) et (4) à la formulation optimale (HLD $=0)$ en fonction du terme ACN.

$\mathrm{ACN}=65,3-10 \mathrm{GN}^{*}(5)$

$\mathrm{ACN}=39,3-6,7 \operatorname{EON}^{*}(6)$

Cette façon d'exprimer l'évolution de l'hydrophilie d'une famille de tensioactifs met en évidence une propriété fondamentale de ces composés. L'ACN calculé à partir des équations (5) et (6) possède en effet un sens physique bien défini. C'est le nombre d'atomes de carbone de l'alcane (ou de l'alcane équivalent) avec lequel on obtient une formulation optimale dans des conditions expérimentales de référence (en général $25^{\circ} \mathrm{C}$, pas d'alcool, pas de sel). Cette valeur de ACN est souvent appelée PACN (alcane préféré) dans la littérature anglo-saxonne. La valeur du PACN calculée par les équations (5) et (6) est une caractéristique, non pas de la phase huile, mais du tensioactif, et permet de le classer sur une échelle absolue apparentée à celle du HLB mais beaucoup plus précise, et mieux définie à la fois expérimentalement et théoriquement. Plus le PACN est élevé, plus le tensioactif est lipophile, et vice versa. Le PACN est identique à la valeur du paramètre caractéristique du tensioactif $(\mathrm{s} / \mathrm{k})$ ou $(\mathrm{a} / \mathrm{k}-\mathrm{EON} / \mathrm{k})$ indiquée sur la figure 7 de la référence [13]. Les équations (5) et (6) sont reportées sur la figure 3 pour visualiser l'évolution de I'hydrophilie des deux familles de tensioactifs en fonction du nombre de motifs hydrophiles. Cette figure fournit deux informations importantes. Tout d'abord, les pentes des droites correspondant aux deux familles de tensioactifs sont différentes, ce qui signifie que les contributions hydrophiles des groupes oxyde d'éthylène et glycérol n'ont pas la même valeur. L'élimination du terme $A C N$ entre les équations (5) et (6) indique en effet qu'un groupe glycérol supplémentaire apporte la même augmentation d'hydrophilie que 1,5 groupes oxyde d'éthylène, ce qui n'a rien de surprenant puisque le groupe hydroxyle supplémentaire compense largement l'effet de l'atome de carbone additionnel, quand on passe d'un motif à l'autre. On peut donc dire que le motif glycérol est $50 \%$ plus hydrophile que le motif oxyde d'éthylène. D'autre part, l'extrapolation des droites de régression jusqu'au tensioactif qui contient un seul motif hydrophile $\left(C_{12} E_{1}\right.$ et $\left.C_{12} G_{1}\right)$ suggère que l'alcool monoéthoxylé $\left(C_{12} E_{1}\right)$ est plus hydrophile que l'ester monoglycérylé $\left(C_{12} G_{1}\right)$, en dépit d'une partie hydrophile plus conséquente (figure 4). Deux raisons peuvent être avancées pour expliquer cette apparente anomalie. D'une part, I'atome d'oxygène de la fonction éther est plus riche en électrons et donc plus enclin à fixer des molécules d'eau par liaison hydrogène que celui de la fonction ester qui est appauvri en électron par l'effet électro-attracteur du groupe carbonyle voisin. D'autre part, les deux fonctions hydroxyles du motif glycérol n'apportent pas une hydrophilie deux fois plus importante que celle du $\mathrm{C}_{12} \mathrm{E}_{1}$ car elles sont certainement engagées dans une liaison hydrogène intramoléculaire (figure 4) qui réduit considérablement leur interaction avec l'eau et donc leur hydrophilie, comme il a été récemment démontré sur des tensioactif à têtes glucosidées [20]. 


\section{Caractérisation d'esters gras}

\section{Alcane équivalent - EACN}

Lorsque la phase huileuse n'est pas constituée d'un alcane linéaire pur, on utilise la notion d'Equivalent Alkane Carbon Number ou EACN. Par définition, I'EACN d'une huile est égal au ACN de l'alcane linéaire qui produit la formulation optimale dans les mêmes conditions expérimentales que I'huile. Le concept du HLD permet de caractériser des huiles plus complexes en déterminant la valeur de leur EACN [13].

\section{Effet de la longueur de chaîne des esters}

Nous avons étudié une série d'esters éthyliques d'acides gras de longueur de chaîne variable. Pour chacune de ces huiles, des balayages unidimensionnels sont réalisés en utilisant GN comme variable de formulation. Les mélanges sont effectués à $55^{\circ} \mathrm{C}$ et contiennent tous $15 \%$ de monolaurate de polyglycérol et des volumes égaux d'huile et d'eau. En ajustant la valeur de GN, on détermine expérimentalement la valeur $\mathrm{GN}^{*}$ pour laquelle la formulation est optimale $(H L D=0)$. Puis, en reportant cette valeur dans l'équation (3), on en déduit l'EACN de l'huile (tableau 1).

Tableau 1. EACN des esters $\mathrm{C}_{n-1} \mathrm{H}_{2 n-1} \mathrm{COOC}_{m} \mathrm{H}_{2 m+1}$

\begin{tabular}{|l|l|l|l|l|l|}
\hline $\mathbf{N}^{\mathbf{0}}$ & Ester & $\mathbf{n}$ & $\mathbf{m}$ & $\mathbf{n}+\mathbf{m}$ & EACN \\
\hline $\mathbf{1}$ & Hexanoate d'éthyle & 6 & 2 & 8 & $-6,5$ \\
\hline $\mathbf{2}$ & Heptanoate d'éthyle & 7 & 2 & 9 & $-0,05$ \\
\hline $\mathbf{3}$ & Octanoate d'éthyle & 8 & 2 & 10 & 8,3 \\
\hline $\mathbf{4}$ & Décanoate d'éthyle & 10 & 2 & 12 & 11,3 \\
\hline $\mathbf{5}$ & Tétradécanoate d'éthyle & 14 & 2 & 16 & 14,0 \\
\hline $\mathbf{6}$ & Heptanoate de méthyle & 7 & 1 & 8 & $-5,6$ \\
\hline $\mathbf{7}$ & Butanoate de butyle & 4 & 4 & 8 & $-6,25$ \\
\hline $\mathbf{8}$ & Propanoate de pentyle & 3 & 5 & 8 & $-6,25$ \\
\hline $\mathbf{9}$ & Acétate d'hexyle & 2 & 6 & 8 & $-3,9$ \\
\hline
\end{tabular}

La figure 5 représente l'évolution comparée de l'EACN des n-alcanes et de celui des esters en fonction du nombre total d'atomes de carbone de l'huile. La variation est strictement linéaire pour les n-alcanes puisque leur EACN est par définition égal à leur ACN. Pour les esters, l'EACN est toujours inférieur à celui de l'alcane correspondant. Cette constatation traduit simplement le fait que les esters sont plus polaires que les alcanes. Par conséquent, la co-solubilisation d'un 
ester avec de l'eau nécessitera un tensioactif plus hydrophile qu'avec l'alcane correspondant. Si I'on observe la courbe d'évolution du EACN des esters en fonction de leur longueur, on voit qu'elle diffère beaucoup de celle des alcanes. Pour les esters les plus courts (hexanoate et heptanoate d'éthyle), I'EACN est très bas en accord avec le caractère polaire très prononcé de ces huiles. En revanche, au fur et à mesure de l'allongement de la chaîne, la polarité apportée par la fonction ester devient moins perceptible et I'EACN des esters se rapproche asymptotiquement de celui des alcanes correspondants. Précisons que lorsque les balayages unidimensionnels sont réalisés en présence de 2-butanol ( $3 \%$ en poids), les résultats expérimentaux sont identiques mais l'équilibre de phase est obtenu plus rapidement car le film interfacial est moins rigide [13]. En revanche, en présence de 1-hexanol, les valeurs des EACN sont non seulement différentes de celles obtenues en absence d'alcool, mais elles sont aussi supérieures à la longueur de chaîne carbonée de l'alcane correspondant [21]. Ce phénomène pourrait être dû à une ségrégation interfaciale du 1-hexanol. Dans ce cas, le co-tensioactif jouerait un double rôle : il diminuerait la rigidité du film interfacial comme le 2-butanol mais il semble qu'il modifierait également l'EACN apparent de l'huile en se concentrant préférentiellement au voisinage de l'interface d'après un mécanisme appelé ségrégation [22] proche de celui attribué à l'effet de l'intermédiaire lipophile (en anglais, lipophilic linker) [23, 24].

\section{Effet de la position de la fonction ester dans la chaîne}

L'influence de la position de la fonction ester de l'huile dans la chaîne carbonée sur la valeur de I'EACN a été étudiée pour une série d'esters gras comprenant un total de 8 atomes de carbone, en détectant la formulation optimale comme précédemment. La figure 6 représente l'évolution de l'EACN en fonction de la position $m$ de la fonction ester dans la chaîne carbonée totale. On constate que les valeurs des EACN d'esters possédant au total 8 atomes de carbone sont négatives quelle que soit la position de la fonction ester dans la chaîne. Elles sont comprises entre -4 et -6 et, par conséquent, toutes inférieures à 8 , valeur du ACN de l'alcane possédant le même nombre d'atomes de carbone. La présence de la fonction ester dans la chaîne de l'huile apporte une polarité notable qui fait chuter I'EACN de 12 unités environ. Ce résultat est en accord avec la littérature [13] qui rapporte que l'oléate d'éthyle possède un EACN de seulement 6,5 en dépit des 20 atomes de carbone présents dans sa structure moléculaire. En revanche, la position de la fonction ester dans la chaîne semble avoir une influence mineure puisque la variation de la valeur du EACN est tout au plus de 2 unités.

\section{Conclusion}

Les monolaurates de polyglycérol, C12Gj, sont des tensioactifs non ioniques présentant des propriétés intéressantes notamment pour des applications en cosmétique. Contrairement aux alcools gras polyéthoxylés, leur comportement de phase ne dépend pas de la température. Ils permettent la formation de microémulsions monophasiques en l'absence de co-tensioactif, probablement grâce à la variété d'espèces chimiques présentes dans le produit commercial, qui introduisent, au niveau du film interfacial, un désordre suffisant pour éviter la formation de mésophases. Le concept du HLD appliqué aux systèmes eau-ester gras-monolaurate de polyglycérol a permis de caractériser d'une part ces tensioactifs et d'autre part, un certain nombre d'huiles de type ester par leur EACN. II apparaît que l'effet du groupe polaire ester domine de plus en plus dominant quand la chaîne apolaire de l'ester devient plus courte. 


\section{RÉFÉRENCES}

1. DOLHAINE H, PREUSS W, WOLLMANN K. Structures in polyglycerol. Fette, Seifen, Anstrichmittel $1984 ; 86,9$ : 339-43.

2. DEBAIG C, BENVEGNU T, PLUSQUELLEC D. Synthesis of linear and cyclic polyglycerols.

Polyglyceryled surfactants : Synthesis and characterization. Oléagineux, Corps Gras, Lipides 2002 ; 9, 2-3 : 155-62.

3. CASSEL $S$, DEBAIG $C$, BENVEGNU T, CHAIMBAULT $P$, LAFOSSE $M$, PLUSQUELLEC $D$, ROLLEN P. Original synthesis of linear, branched and cyclic oligoglycerol standards. Eur. J. Org. Chem. 2001 ; $5: 875-96$.

4. SAAD H. J. Am. Oil Chem. Soc. $1975 ;$ 52, 127A.

5. HEMKER W. Associative structures of polyglycerol esters in food emulsions. J. Am. Oil Chem. Soc. $1981 ; 58,2: 114-9$.

6. PROSERPIO G. Poligliceroli in luogo di polietilenglicoli, Evoluzione in emulsologia, COSNDG $2000 ; 112,97: 38$.

7. SALAGER JL (1999). Microemulsions, in Handbook of Detergents - Part A : Properties, Broze G Ed., M. Dekker, New York, pp. 253-302.

8. KUNIEDA $H$, NAKANO A, AKIMARU M. The effect of mixing of surfactants on solubilization in a microemulsion system. J. Colloid. Interface Sci. $1995 ; 170$ (1) : 78-84.

9. ISHITOBI M, KUNIEDA HH. Effect of chain length distribution on the phase behavior of polyglycerol fatty acid ester in water. Colloid Polym. Sci. 2000 ; 278, 9 : 899-904.

10. SALAGER JL (2000). Formulation concepts for the emulsion maker, Drugs and Pharmaceutical Sciences, 105, 19-72, Marcel Dekker, New-York.

11. SALAGER JL (2000). Emulsion properties and related know-how to attain them, Drugs and Pharmaceutical Sciences, 105, 73-125, Marcel Dekker, New-York.

12. NARDELLO-RATAJ V, POPRAWSKI J, CHAILLOUX N, AUBRY JM. Micro- and macroemulsification of cosmetic oils by the " optimum formulation " concept. Comunicaciones presentadas a las Jornadas del Comite Espanol de la Detergencia $2002 ; 32: 143-52$.

13. SALAGER JL, ANTON RE, ANDEREZ JM, AUBRY JM (2001). Formulation des microémulsions par la méthode du HLD. Techniques de l'ingénieur, Génie des procédés, N J2155, 1-20.

14. POPRAWSKI J, MARTI MJ, SALAGER JL, AUBRY JM. Application of the HLD formulation concept to microemulsions containing pine oil and a non-ionic surfactant. Polym. Int. $2003 ;$ 52, 4 : 629-32.

15. NARDELLO V, CHAILLOUX N, POPRAWSKI J, SALAGER JL, AUBRY JM. HLD concept as a tool for the characterization of cosmetic hydrocarbon oils. Polym. Int. $2003 ; 52,4: 602-09$. 
16. HUH C. Interfacial tensions and solubilizing ability of a microemulsion phase that coexists with oil and brine. J. Colloid. Interface Sci. 1979 ; 71 : 408-26.

17. De MeUlenaer B, VAN ROYen G, VANHOUtTE B, huYgheBAert A. Combined liquid and gas chromatographic characterisation of polyglycerol fatty acid esters. J. Chromatography $A 2000$; $896: 239-51$.

18. CASSEL $S$, CHAIMBAULT P, DEBAIG C, BENVEGNU T, CLAUDE $S$, PLUSQUELLEC D, ROLLIN P, LAFOSSE M. Liquid chromatography of polyglycerol fatty esters and fatty ethers on porous grahitic carbon and octadecyl silica by using evaporative light scattering detection and mass spectrometry. J. Chromatography A 2001 ; 919 : 95-106.

19. KUNIEDA H, AKAHANE A, FENG J, ISHITOBI M. Phase behavior of polyglycerol didodecanoates in water. J. Colloid. Interface Sci. $2002 ; 245,2$ : 365-70.

20. GOETHALS G, FERNANDEZ A, MARTIN P, MINANA-PEREZ M, SCORZZA C, VILLA P, GODE P. Spacer arm influence on glucido-amphiphilic compound properties. Carbohydrate Polymers $2001 ; 45,2$ : 147-54.

21. NARDELLO-RATAJ V, POPRAWSKI J, CHAILLOUX N, AUBRY JM (2003). Application of the HLD concept to the characterization of polyglycerol ester surfactants, Comunicaciones presentadas a las Jornadas del Comite Espanol de la Detergencia $2003 ; 33: 49-58$.

22. GRACIAA A, LACHAISE J, CUCUPHAT C, BOURREL M, SALAGER JL. Interfacial segregation of an ethyl oleate/hexadecane oil mixture in microemulsion systems. Langmuir1993 ; 9, $6: 1473-8$.

23. GRACIAA A, LACHAISE J, CUCUPHAT C, BOURREL M, SALAGER JL. Improving solubilization in microemulsions with additives - Part I : The lipophilic linker role. Langmuir $1993 ; 9:$ 669-72.

24. GRACIAA A, LACHAISE J, CUCUPHAT C, BOURREL M, SALAGER JL. Improving solubilization in microemulsions with additives - Part II : Long chain alcohol as lipophilic linkers. Langmuir 1993 ; $9: 3371-4 . n$ 
Illustrations

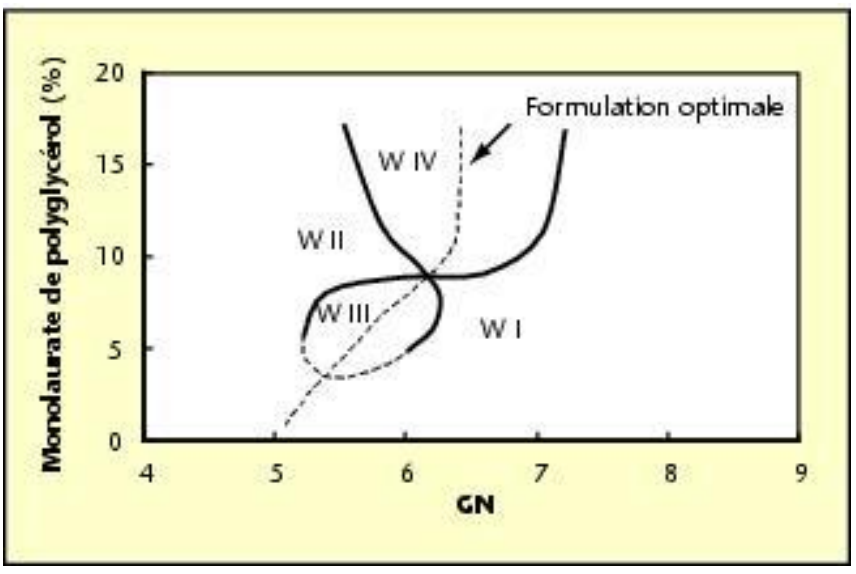

Figure 1. Comportement de phase du système eau-dodécane-monolaurate de polyglycérol-hexanol ( $3 \%$ en poids) en fonction de la concentration en tensioactif et du nombre d'unités glycérol. Conditions : $T=55^{\circ} \mathrm{C}$, eau/dodécane $=1$ (en volume).

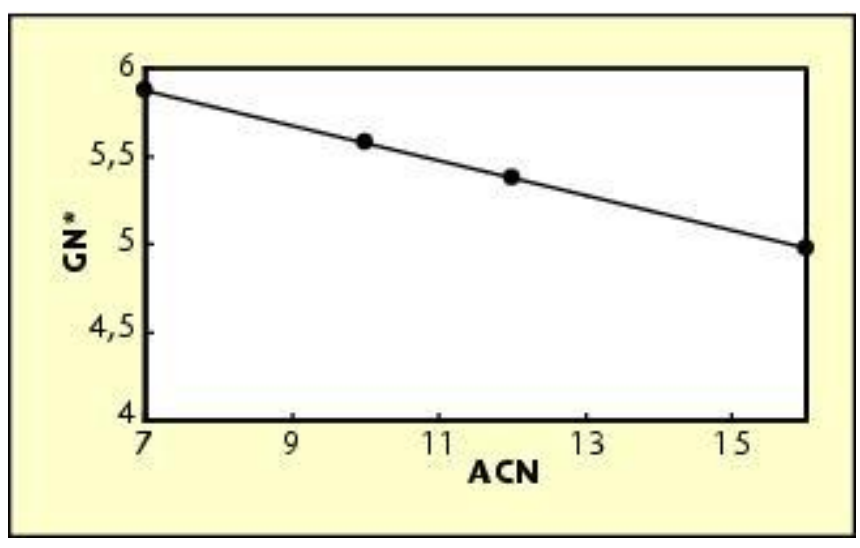

Figure 2. Évolution du nombre de motifs glycérol à la formulation optimale pour différents alcanes linéaires. Conditions : [monolaurate de polyglycérol] = $15 \%$ (en poids), eau/huile = 1 (en volume), $T=55^{\circ} \mathrm{C}$.

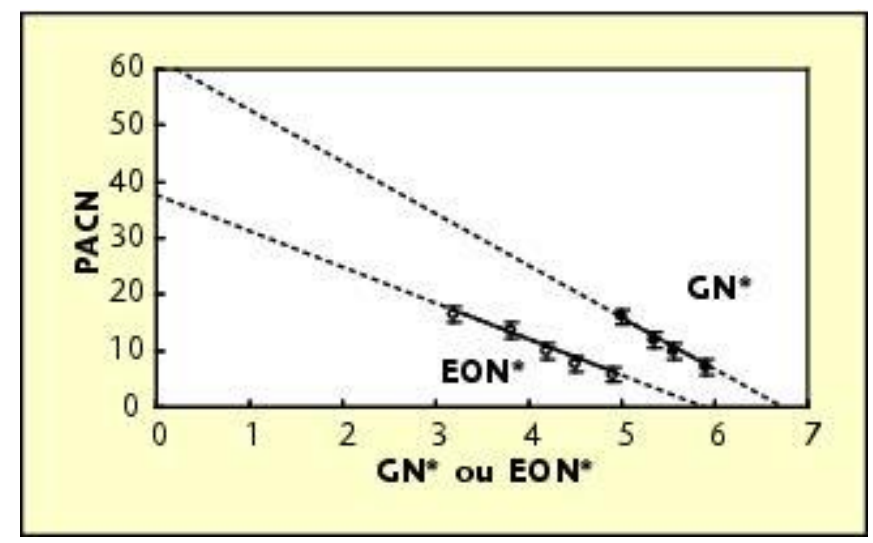

Figure 3. Évolution du PACN des tensioactifs $C_{12} E_{j}$ et $C_{12} G_{j}$ en fonction du nombre de motifs hydrophiles éthoxylés ou glycérolés. 


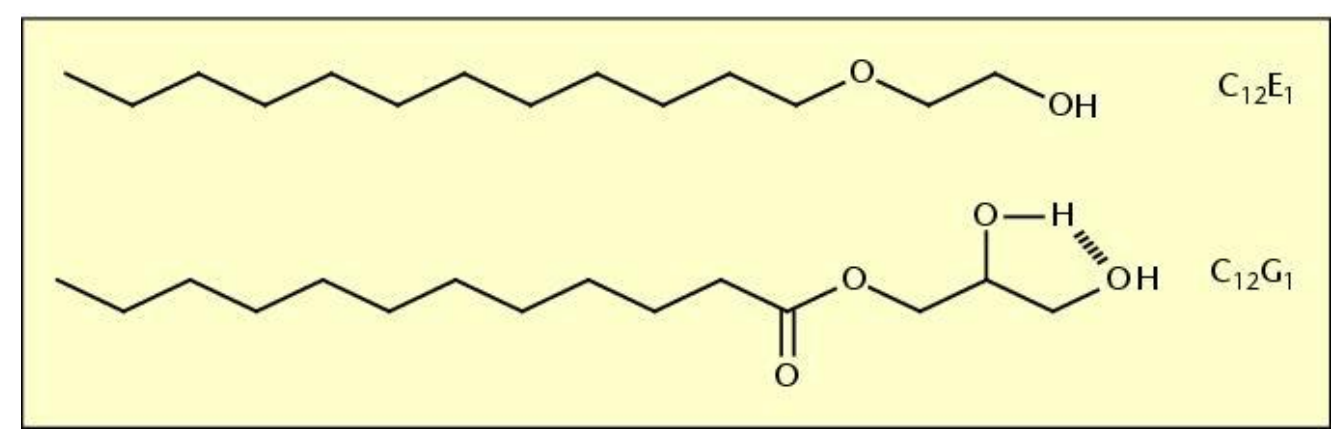

Figure 4. Structures moléculaires de l'alcool laurique monoéthoxylé $C_{12} E_{1}$ et du lauryl ester de monoglycérol $C_{12} G_{1}$.

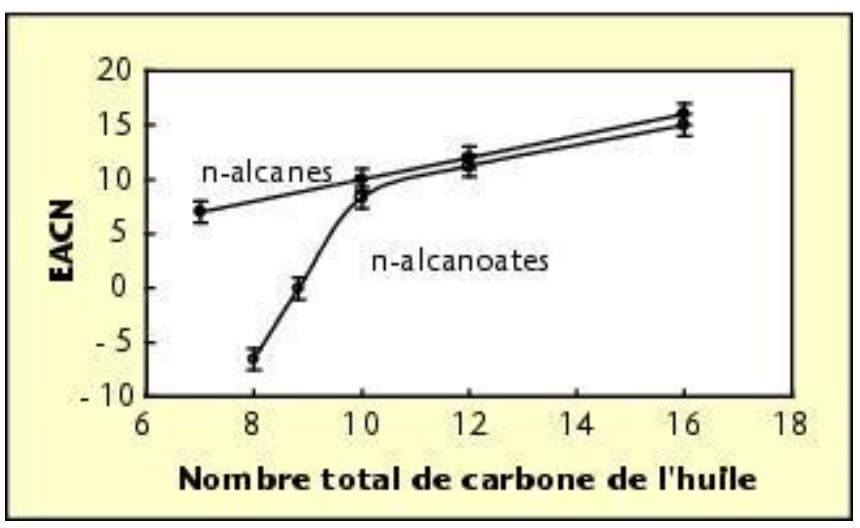

Figure 5. Evolution des EACN des n-alcanes $(\bullet)$ et des $n$-alcanoates d'éthyle $(\bullet)$ en fonction du nombre total d'atomes de carbone de l'huile.

Conditions : $C_{12} G_{j}=15 \%$ en poids, eau/ester $=1$ (en volume), $T=55^{\circ} \mathrm{C}$.

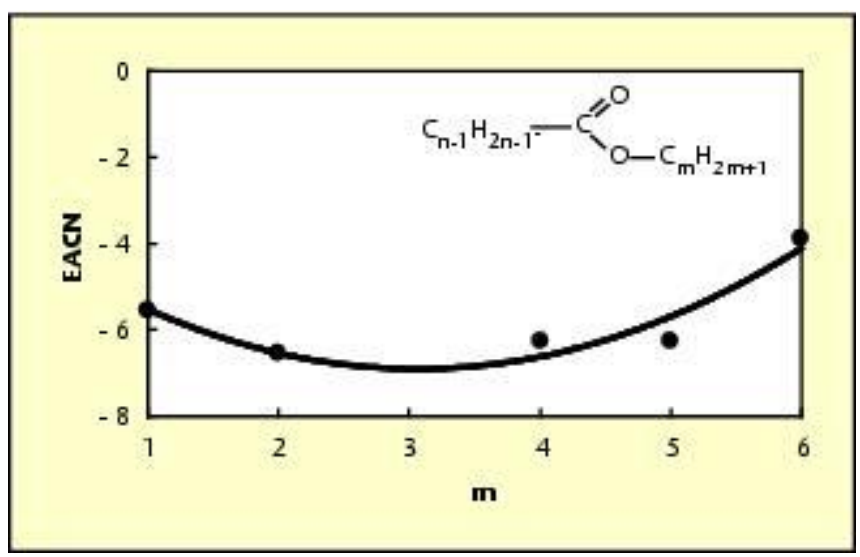

Figure 6. Évolution de l'EACN d'esters comportant 8 atomes de carbone au total en fonction de la position de la fonction ester $(5<x<0)$. Conditions : [monolaurate de polyglycérol] $=15 \%$ (en masse), eau/ester $=1$ (en volume), $T=55^{\circ} \mathrm{C}$. 